\title{
Influence of shape fluctuations in relativistic heavy ion collisions
}

\author{
A. Rosenhauer and H. Stöcker \\ Institut für Theoretische Physik, Johann Wolfgang Goethe-Universität, 6000 Frankfurt am Main 11, \\ Federal Republic of Germany
}

\author{
J. A. Maruhn* and W. Greiner* \\ Joint Institute for Heavy Ion Research, Holifield Heavy Ion Research Facility, Oak Ridge, Tennessee 37831
}

(Received 25 February 1986)

\begin{abstract}
The influence of fluctuations of the shape degree of freedom in collisions of deformed nuclei with energies between 0.8 and $2.1 \mathrm{GeV} /$ nucleon is analyzed on the basis of an intranuclear cascade simulation for the strongly deformed systems ${ }^{46} \mathrm{Ti}+{ }^{46} \mathrm{Ti}$ and ${ }^{166} \mathrm{Er}+{ }^{166} \mathrm{Er}$. While there is a considerable sensitivity of the global event variables to the orientation for polarized beams and targets, this dependence disappears in the average over all orientations for impact parameter selected and integrated events. The dependence of the nuclear stopping and thermalization on the size of the system under consideration and on the bombarding energy is also investigated.
\end{abstract}

\section{INTRODUCTION}

We recently proposed ${ }^{1}$ to investigate polarized shape orientations in central heavy-ion collisions at relativistic energies. This study is motivated by successful experimental verification of collective flow effects ${ }^{2}$ predicted early in the fluid dynamical model, ${ }^{3}$ in which the incoming nuclei are assumed to be spheres of nuclear matter with a homogeneous distribution of nucleons inside. Taking into account that many nuclei have nonspherical shapes even in their ground state, it appears advisable to point out how far the simplifying assumption of spherical nuclei instead of deformed ones affects the experimentally available observables.

In an experiment with nonpolarized beams and targets one deals with a large number of reactions so that the measurement can be interpreted as an ensemble average over collisions between deformed nuclei with different orientations which smear out the effects of deformation. From this point of view shape deformations can be considered as a fluctuation of the spherical shape. In this paper we want to study the effects of impact parameter averaging and orientation averaging which allow direct comparison to experimental data and propose a method with which the forecast polarization effects could be studied.

After a discussion of the results obtained from central collisions with polarized beams and targets in Sec. II, we discuss the effects on observables if the selection of central reactions is given up in Sec. III. Further insight into the reaction mechanism is obtained from an orientation average of central collisions in Sec. IV. In Sec. $\mathrm{V}$ the experimental situation is simulated by averaging both over the orientations and over a range of impact parameters. The summary and concluding remarks are given in Sec. VI.

\section{CENTRAL COLLISIONS OF DEFORMED POLARIZED NUCLEI}

The relativistic collision process is described microscopically using the intranuclear cascade simulation program of Cugnon et al. ${ }^{4}$ We consider collisions of the symmetric systems ${ }^{46} \mathrm{Ti}+{ }^{46} \mathrm{Ti}$ and ${ }^{166} \mathrm{Er}+{ }^{166} \mathrm{Er}$ which exhibit strong ground state quadrupole deformations $\left(\beta_{2}=0.28\right.$ and 0.31 , respectively $\left.{ }^{5,6}\right)$ at relativistic energies $(0.8$ and $2.1 \mathrm{GeV} /$ nucleon).

Two extreme relative orientations are studied, namely the case of a "head on head" collision ( $\mathrm{HOH})$, where the direction of the maximum elongation of both nuclei coincides with that of the beam axis [Fig. 1(a)], and the case "belly on belly" (BOB), where the "heads" of both nuclei from the $\mathrm{HOH}$ constellation have been rotated by an angle of $\theta=90^{\circ}$ in the plane perpendicular to the beam axis [Fig. 1(b)]. The systematic influence of the deformation for these two extreme cases is obtained by comparing with a reference calculation for two spherical nuclei (SOS) [Fig. $1(\mathrm{c})]$.

We consider the following characteristic observables:

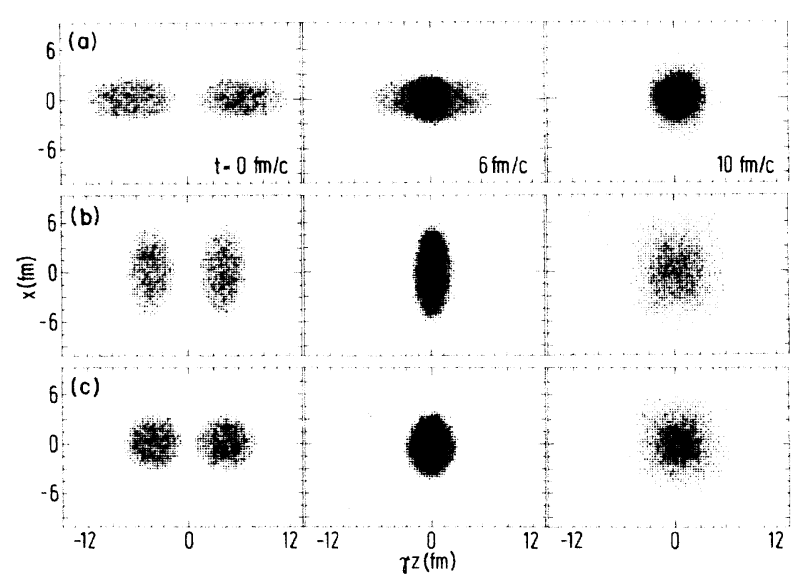

FIG. 1. Illustration of the compression and expansion stage in a central collision. The density at different reaction times as indicated is plotted for the three constellations. The head on head collision corresponds to (a), the belly on belly orientation to (b), and the reaction of two spherical nuclei $\left({ }^{46} \mathrm{Ti}+{ }^{46} \mathrm{Ti}\right.$ at 2.1 $\mathrm{GeV} /$ nucleon) is illustrated in (c). 
(1) the maximum compression $\rho / \rho_{0}$ obtained during the course of the collision in the central region of the reaction (i.e., the number of particles in a sphere in the center of the c.m. frame with radius $R=2 \mathrm{fm}$ );

(2) the number of nucleon-nucleon collisions per reaction $N_{\text {coll }}$;

(3) the number of pions $N_{\pi}$ at the breakup time ( $t \simeq 15$ $\mathrm{fm} / \mathrm{c})$;

(4) the number of particles detected by the plastic ball detector $N_{\text {mul }}$;

(5) the global variables aspect ratio $R_{13}$; and

(6) the flow angle $\theta_{F}$ defined in the c.m. frame. ${ }^{7,8}$

All results presented here are obtained by $1000(500)$ intranuclear cascade simulations for the system ${ }^{46} \mathrm{Ti}+{ }^{46} \mathrm{Ti}$ $\left({ }^{166} \mathrm{Er}+{ }^{166} \mathrm{Er}\right)$, respectively. They have been performed on the AP164 processor at Gesellschaft für Schwerionenforschung (GSI), with a run time for ${ }^{46} \mathrm{Ti}$ of $<3$ sec per event. All observables discussed above have been listed for the cases BOB, HOH, and SOS in Table I.

The most significant differences for central collisions of polarized beams and targets occur in the mean flow angle $\theta_{F}$. Considering the distribution of $\theta_{F}$ and its mean value for all three constellations, this variable seems appropriate to discriminate $\mathrm{HOH}$ collisions from all other cases. ${ }^{1}$

The $\mathrm{HOH}$ collision seems to be most equilibrated in comparison to all other cases resulting in the largest collision numbers, the smallest aspect ratio but the largest flow angles, the highest densities and the largest pion numbers. This is understandable in terms of the increase of the effective target thickness in this constellation.

We observe a significant change in transparency within the considered systems: in the case of BOB collisions we obtain more spectators than in the spherical case, while in $\mathrm{HOH}$ reactions less spectators are found.

The apparent decrease of spectators in the $\mathrm{HOH}$ configuration is a consequence of the favorable geometry, as in such collisions each individual projectile nucleon has to pass through a larger spatial region of target gas (elongation along the beam axis) than in the case of a collision of two spherical nuclei (see Fig. 1). The same argument also applies to the BOB constellations, where fewer collisions are expected. The SOS case finally lies in between the values obtained for the two extreme orientations.

Note that the occurrence of multiple collisions suffered by one single nucleon is confirmed by the fact that $N_{\text {coll }}$ as a function of the mass number $A$ increases much faster than $A^{2 / 3}$ which is expected from geometric single scattering considerations. Although the mean number of collisions per nucleon is larger by a factor of 2 for the heavier system [at $2.1 \mathrm{GeV} /$ nucleon, $\left\langle N_{\text {coll }}\right\rangle /$ nucleon $=4.7$ for ${ }^{46} \mathrm{Ti}$ (SOS), $\left\langle N_{\text {coll }}\right\rangle /$ nucleon $=8.5$ for ${ }^{166} \mathrm{Er}$ (SOS)], multiple scattering is already observed in the relatively small system ${ }^{46} \mathrm{Ti}+{ }^{46} \mathrm{Ti}$.

In contrast to the hydrodynamic scenario where the scaling properties predict a maximum density independent of the mass of the colliding nuclei, ${ }^{9}$ the intranuclear cascade (INC) simulation forecasts a strong increase of the compression with the mass of the system: $\rho / \rho_{0}=3.9$ for ${ }^{46} \mathrm{Ti}(\mathrm{SOS})$ at $E_{\mathrm{lab}}=2.1 \mathrm{GeV} /$ nucleon to $\rho / \rho_{0}=4.8$ for ${ }^{166} \mathrm{Er}$ (SOS) at the same projectile energy. The strong dependence of the compression on the mass number clearly reflects the increased stopping power of heavy systems.

At the same time we observe a substantial increase of the maximum density with increasing beam energy as one would expect from hydrodynamic calculations: for the system ${ }^{166} \mathrm{Er}(\mathrm{SOS}) \rho / \rho_{0}$ increases from 4.2 at $E_{\mathrm{lab}}=0.8$ $\mathrm{GeV} /$ nucleon to $\rho / \rho_{0}=4.8$ at $E_{\text {lab }}=2.1 \mathrm{GeV} /$ nucleon.

Concerning the dependence of $\theta_{F}$ on the mass of the system we would like to note that there is a strong increase of $\theta_{F}$ with $A$ [see, e.g., ${ }^{46} \mathrm{Ti}$ (BOB) at 0.8 $\mathrm{GeV} /$ nucleon from $33^{\circ}$ to $52^{\circ}$ for ${ }^{166} \mathrm{Er}(\mathrm{BOB})$ at the same energy]. This provides further indication for the strongly increased stopping power with increasing $A$, which is also reflected in the above-mentioned strong increase of the maximum compression. These effects are indicative of an

TABLE I. Characteristic observables calculated for the cases $\mathrm{HOH}, \mathrm{BOB}$, and SOS for the systems ${ }^{46} \mathrm{Ti}+{ }^{46} \mathrm{Ti}$ and ${ }^{166} \mathrm{Er}+{ }^{166} \mathrm{Er}$ at the projectile energy $E_{\mathrm{p}}$ as indicated: the quantity $\rho / \rho_{0}$ is the maximum compression obtained in the course of the collision in the central region of the reaction, $N_{\text {coll }}$ is the number of binary collisions per reaction, $N_{\pi}$ the number of produced pions, $N_{\text {mul }}$ the multiplicity detected by the plastic ball, $R_{13}$ the aspect ratio, and $\theta_{F}$ the flow angle. For central collisions of polarized beams and targets the most significant differences occur in the mean flow angle $\theta_{F}$, which seems to be appropriate to discriminate HOH collisions from all other cases.

\begin{tabular}{|c|c|c|c|c|c|c|c|c|c|}
\hline & $E_{\mathrm{p}}$ & & $\rho / \rho_{0}$ & $N_{\text {coll }}$ & $N_{\pi}$ & $N_{\text {mul }}$ & $R_{13}$ & $\theta_{F}$ & $E_{T}$ \\
\hline \multirow[t]{6}{*}{${ }^{46} \mathrm{Ti}$} & 2.1 & $\mathrm{HOH}$ & 3.9 & 454 & 30.9 & 89.2 & 1.91 & 35.8 & 11.4 \\
\hline & & BOB & 3.7 & 420 & 30.9 & 87.9 & 2.14 & 18.8 & 10.7 \\
\hline & & SOS & $3.9 \pm 0.1$ & $435 \pm 1$ & $30.9 \pm 0.1$ & $88.4 \pm 0.1$ & $2.03 \pm 0.01$ & $24.6 \pm 0.4$ & $10.9 \pm 0$ \\
\hline & 0.8 & $\mathbf{H O H}$ & 3.4 & 410 & 11.3 & 87.7 & 1.84 & 50.3 & 5.0 \\
\hline & & BOB & 3.4 & 387 & 11.6 & 86.2 & 1.81 & 33.2 & 4.7 \\
\hline & & SOS & 3.5 & 398 & 11.3 & 86.7 & 1.81 & 38.8 & 4.8 \\
\hline \multirow[t]{6}{*}{${ }^{166} \mathrm{Er}$} & 2.1 & $\mathrm{HOH}$ & 4.8 & 2936 & 105.3 & 327.0 & 1.52 & 55.4 & 43.8 \\
\hline & & BOB & 4.6 & 2863 & 108.7 & 324.1 & 1.61 & 20.8 & 40.6 \\
\hline & & SOS & $4.8 \pm 0.1$ & $2811 \pm 3$ & $107.4 \pm 0.3$ & $325.4 \pm 0.1$ & $1.47 \pm 0.01$ & $33.1 \pm 0.5$ & $41.6 \pm 0.1$ \\
\hline & 0.8 & $\mathrm{HOH}$ & 4.1 & 2542 & 34.5 & 323.1 & 1.71 & 67.0 & 19.1 \\
\hline & & BOB & 4.1 & 2396 & 36.2 & 318.3 & 1.43 & 52.3 & 17.6 \\
\hline & & SOS & 4.2 & 2485 & 35.2 & 320.1 & 1.45 & 55.4 & 18.2 \\
\hline
\end{tabular}


TABLE II. Same observables as in Table I but for impact parameter averaged collisions of deformed polarized nuclei. The large differences in the flow angles obtained from central collisions have been diminished but the systematics of the change within the constellations is just the same as for central collisions.

\begin{tabular}{|c|c|c|c|c|c|c|c|c|}
\hline & $E_{\mathrm{p}}$ & & $\rho / \rho_{0}$ & $N_{\text {coll }}$ & $N_{\pi}$ & $N_{\text {mul }}$ & $R_{13}$ & $\theta_{F}$ \\
\hline \multirow[t]{6}{*}{${ }^{46} \mathrm{Ti}$} & 0.8 & BOB & 2.6 & 309 & 9.0 & 76.4 & 3.5 & 17.4 \\
\hline & & HOH & 2.5 & 298 & 7.8 & 74.8 & 4.2 & 22.4 \\
\hline & & SOS & 2.6 & 300 & 8.2 & 74.8 & 4.1 & 19.2 \\
\hline & 2.1 & BOB & 2.9 & 341 & 24.3 & 78.8 & 4.2 & 13.0 \\
\hline & & НОН & 2.7 & 334 & 22.1 & 77.1 & 5.1 & 17.9 \\
\hline & & SOS & $2.9 \pm 0.2$ & $336 \pm 5$ & $22.7 \pm 0.5$ & $77.7 \pm 0.6$ & $4.8 \pm 0.2$ & $15.4 \pm 0.7$ \\
\hline \multirow[t]{3}{*}{${ }^{166} \mathrm{Er}$} & 2.1 & BOB & 3.6 & 2112 & 82 & 294 & 3.7 & 13.1 \\
\hline & & $\mathrm{HOH}$ & 3.4 & 2034 & 70 & 289 & 4.9 & 19.9 \\
\hline & & SOS & $3.5 \pm 0.3$ & $2070 \pm 28$ & $76 \pm 2$ & $293 \pm 2$ & $4.2 \pm 0.3$ & $17.0 \pm 0.6$ \\
\hline
\end{tabular}

enhanced equilibration in the heavier system. The decrease of $\theta_{F}$ with $E_{\text {lab }}$ from $50^{\circ}$ at $0.8 \mathrm{GeV} /$ nucleon to $36^{\circ}$ at $2.1 \mathrm{GeV} /$ nucleon for ${ }^{46} \mathrm{Ti}(\mathrm{HOH})$ is the result of an increased weight of the spectators because of their higher incident momenta.

\section{IMPACT PARAMETER AVERAGED COLLISIONS OF DEFORMED NUCLEI}

Let us now discuss the influence of impact parameter averaging. A range of impact parameters up to halfnuclear radius is taken randomly with the appropriate geometric weight. Peripheral collisions are excluded because fluctuations grow very fast at large impact parameters, i.e., when a decreasing number of particles is involved in the reaction.

The results of the calculations are listed in Table II. The dramatic (factor of 2) differences in flow angles obtained for central collisions have been diminished to about $20 \%$. The systematics of the change is the same as for central collisions.

One essential feature of noncentral collisions is the fact that in comparison to the $\mathrm{HOH}$ reaction, the $\mathrm{BOB}$ configuration is related to larger reaction cross sections unless the vector pointing in the direction of maximum elongation is perpendicular to the reaction plane. Obviously the smaller reaction cross section of the $\mathrm{HOH}$ constellation is balanced by an increased number of collisions per participating nucleon. This geometrical argument is confirmed by finding that the same number of collisions occur in the $\mathrm{HOH}$ and $\mathrm{BOB}$ reaction, while in the $\mathrm{HOH}$ collisions one observes approximately $10 \%$ more spectators and in general greater widths of all observables.

Thus the influence of two competing mechanisms on the observables has to be considered. HOH reactions are characterized by more violent collisions at small impact parameters, whereas in the BOB case a more transparent reaction in a greater reaction volume mainly contributes at intermediate impact parameters. With the argument of more participating particles suffering a smaller number of collisions for BOB reactions we also can explain the considerable increase of pions which are created at large relative momenta within the first two collisions of each participating nucleon. The same holds for the decrease in the

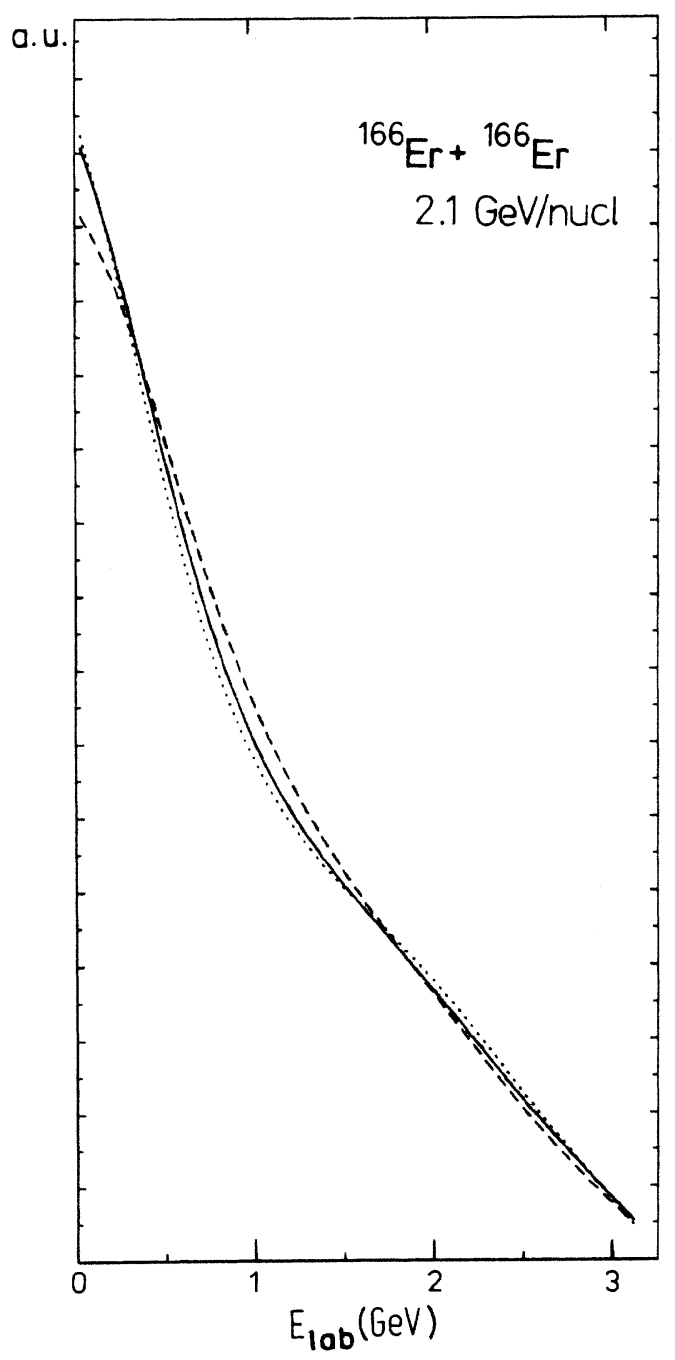

FIG. 2. Differential cross section $d N / d E$ of all participant particles detected by the plastic ball in the laboratory system. The inclusive spectrum is obtained from reactions between spherical nuclei (full line) and from reactions with deformed polarized nuclei: The dotted line corresponds to the $\mathrm{HOH}$ orientation, the dashed line to the BOB case. We do not observe drastic differences within the constellations under consideration. 
aspect ratio, which is far away from the thermal limit so that even nucleons which have been scattered only once will decrease $R_{13}$.

The differential cross section $d N / d E$ of all participant particles detected by the plastic ball in the laboratory system has been plotted for the cases HOH, BOB, and SOS in Fig. 2. The deviations within the range of good statistics are less than $5 \%$ and thus become comparable to the uncertainties of the Monte Carlo simulation.

It is interesting to mention that for all cases the standard deviation of the distribution of the flow angles $\theta_{F}$ becomes smaller by about $30-50 \%$ due to the impact parameter averaging. Apparently, the width of the distribution of $\theta_{F}$ depends strongly on the impact parameter. The standard deviation as a function of $\theta_{F}$ shows a maximum for central and almost central collisions and tends to zero because of the increasing background of spectators and singly collided particles. Hence we have a contribution of a large standard deviation from the small range of central collisions and one of small standard deviation from noncentral collisions dominating due to their statistical weight.

The standard deviation of most other listed observables increases signficantly in comparison to central collisions (compare the standard deviation in Tables I and II for the reaction ${ }^{46} \mathrm{Ti}+{ }^{46} \mathrm{Ti}$ at $2.1 \mathrm{GeV} /$ nucleon).

At a projectile energy of $0.8 \mathrm{GeV} /$ nucleon the same behavior in observables, cross sections, and standard deviation is revealed. The deviations from the spherical case SOS are in the same order of magnitude as at 2.1 $\mathrm{GeV} /$ nucleon.

\section{CENTRAL COLLISIONS OF NONPOLARIZED DEFORMED NUCLEI}

Up to now, we have only considered reactions of nuclei with deformed shapes at two particular relative orientations, namely $\mathrm{HOH}$ and BOB. Although these studies yield some important insight into how geometry influences the observables, they are of no practical interest unless the orientation of the incident nuclei is exactly known: we do not know the consequence of a rotation of one nuclei with respect to the other such as "belly on head" or a rotation of the two symmetry axes of each nuclei out of the reaction plane.

In order to investigate the effects of random orientations of target and projectile, both nuclei are rotated by

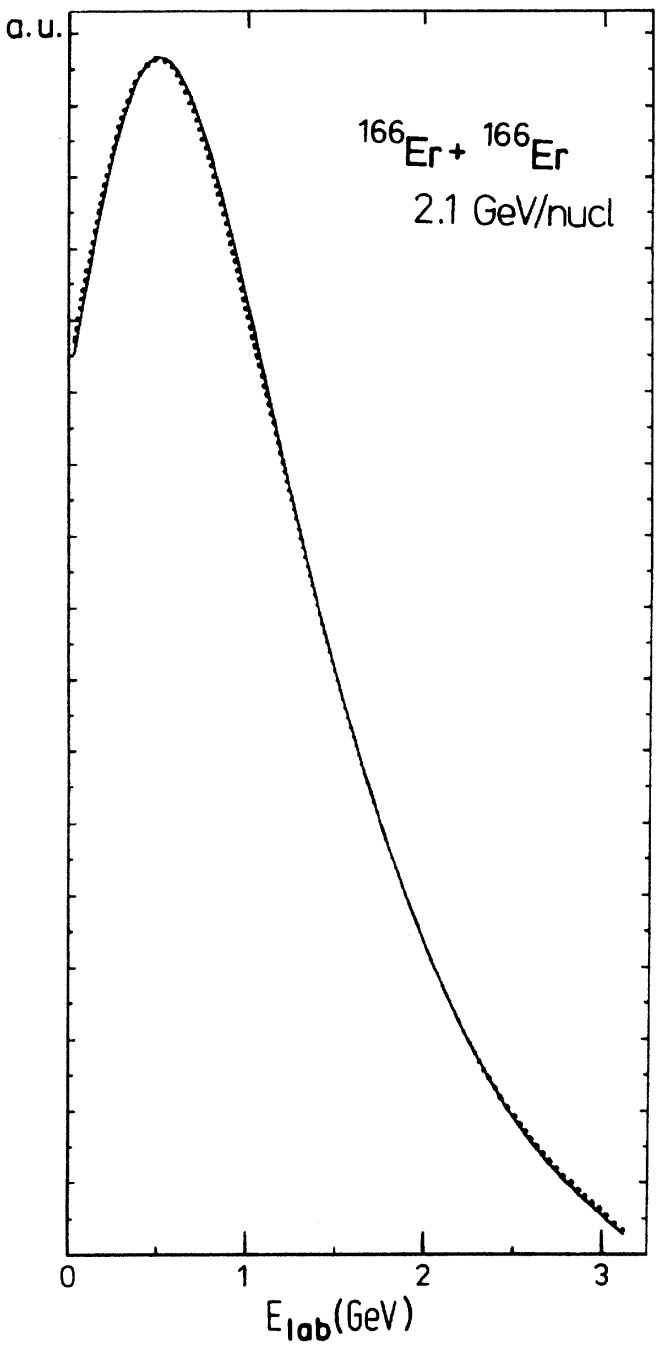

FIG. 3. Differential cross section $d N / d E$ of participant particles from central collisions of spherical nuclei (full line) and from reactions with deformed nuclei at random orientations (dotted line). Both reactions can be considered practically equivalent.

two Euler angles, which are determined randomly with the corresponding geometrical weight.

In Table III we compare the results obtained from a central collision of two spherical nuclei (case $S$ ) with the results from a central reaction between two randomly oriented deformed nuclei (case Av).

TABLE III. Results obtained from central collisions of deformed nuclei at random orientations (case Av) and from central collisions of spherical nuclei (case $S$ ). Note the statistically significant $10 \%$ decrease in $\theta_{F}$ when going from spherical to the randomly orientated deformed case.

\begin{tabular}{|c|c|c|c|c|c|c|c|c|}
\hline & $E_{\mathrm{p}}$ & & $\rho / \rho_{0}$ & $N_{\text {coll }}$ & $N_{\pi}$ & $N_{\text {mul }}$ & $R_{13}$ & $\theta_{F}$ \\
\hline \multirow[t]{4}{*}{${ }^{46} \mathrm{Ti}$} & 0.8 & Av & 2.8 & 394 & 11.4 & 88.6 & 1.78 & 38.7 \\
\hline & & $S$ & 2.8 & 398 & 11.4 & 86.7 & 1.81 & 38.7 \\
\hline & 2.1 & Av & 3.1 & 430 & 30.9 & 88.2 & 2.06 & 22.8 \\
\hline & & $S$ & $3.1 \pm 0.2$ & $436 \pm 1$ & $30.7 \pm 0.2$ & $88.5 \pm 0.1$ & $2.04 \pm 0.02$ & $24.9 \pm 0.6$ \\
\hline \multirow[t]{2}{*}{${ }^{166} \mathrm{Er}$} & 2.1 & Av & 3.7 & 2743 & 107.2 & 325.5 & 1.53 & 28.9 \\
\hline & & $S$ & $3.9 \pm 0.2$ & $2817 \pm 4$ & $106.8 \pm 0.4$ & $325.7 \pm 0.2$ & $1.47 \pm 0.01$ & $32.4 \pm 0.5$ \\
\hline
\end{tabular}


As is to be expected, the dramatic differences in the flow angle $\theta_{F}$, which allowed one to distinguish between the constellations $\mathrm{HOH}, \mathrm{BOB}$, and SOS have been drastically diminished as a result of the orientation average. Still, one should note that $\theta_{F}$ is decreased by about $10 \%$ when deformed nuclei are studied as compared to spherical nuclei of the same mass. This prediction could be checked experimentally.

In general, the dynamics of the reaction at random orientation seems to be somewhat more transparent than in case $S$ : besides a decrease in the number of collisions we obtain a smaller compression, worse degradation of the initial momenta, and smaller flow angles. All values for the observables under consideration lie in between the intervals which are defined by two extreme cases $\mathrm{HOH}$ and BOB and approach the values obtained from the reaction of two spherical nuclei and deviations of less than $10 \%$.

The width of the distributions increases slightly due to the averaging procedure, especially that of the flow angle. However this increase is only about $10 \%$ compared to central collisions of spherical nuclei.

Finally, the energy spectrum $d N / d E$ proves that the orientation averaging and the SOS reactions can be considered to be practically equivalent. It is interesting that the energy spectra for central collisions are peaked at the c.m. energy ( $\simeq 0.4 \mathrm{GeV} /$ nucleon, see Fig. 3 ), while the impact parameter averaging results in a peak at $E=0$. This clearly demonstrates that almost complete stopping occurs in central collisions. Again, qualitatively analogous results are found at a beam energy of $0.8 \mathrm{GeV} /$ nucleon.

\section{REACTIONS OF NUCLEI WITH DEFORMED SHAPES AT RANDOM ORIENTATIONS AND IMPACT PARAMETERS}

The present experimental situation (nonavailability of polarized beams and targets) is best simulated by averaging over all possible orientations of deformed nuclei as well as over impact parameters. The results of such an ensemble average (case Av) are compared to the impact parameter averaged reaction of two spherical nuclei in Table IV.

Let us remember the tendency of the results from the impact parameter averaged ensemble (Sec. III) and those from the average over orientations at central collisions in the last section: in both cases the observables tend towards the results obtained from the corresponding reac-

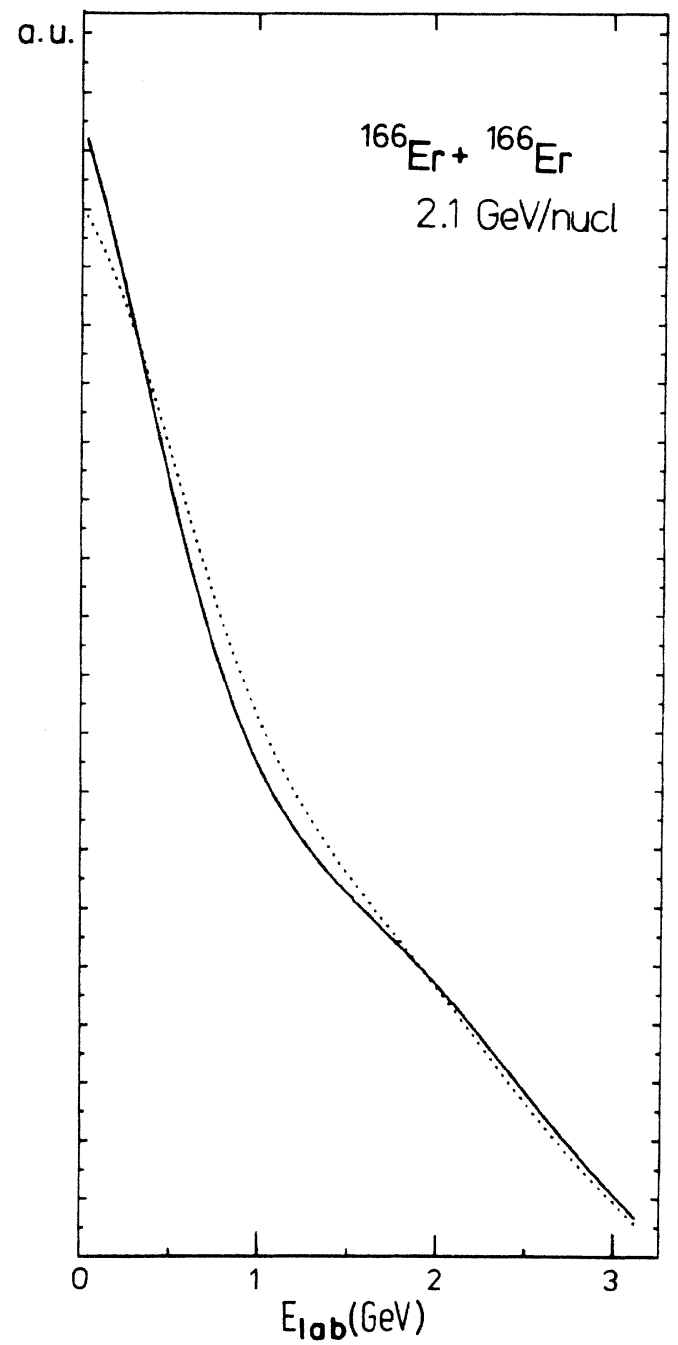

FIG. 4. Differential cross section $d N / d E$ from reactions between spherical nuclei (full line) and from reactions with deformed nuclei at random orientations (dotted line) including impact parameter averaging. Only slight differences between the spherical and randomly rotated cases are detected.

tion of two spherical nuclei. As to be expected, averaging over the five free parameters $\left(b, \theta_{1}, \phi_{1}, \theta_{2}, \phi_{2}\right)$ results in a de facto disappearance of all differences between observables in reactions of deformed and spherical nuclei, respectively. There is one exception, namely the pion yield: the higher number of $\mathrm{N}-\mathrm{N}$ collisions results in an

TABLE IV. Impact parameter averaged results for the observables considered in Table I for reactions of spherical nuclei (case $S$ ) and from reactions averaged over orientation (case Av). The averaging results in a de facto disappearance of all differences between observables in reactions of deformed and spherical nuclei, respectively.

\begin{tabular}{ccccccccc}
\hline \hline & $E_{\mathrm{p}}$ & & $\rho / \rho_{0}$ & $N_{\text {coll }}$ & $N_{\pi}$ & $N_{\text {mul }}$ & $R_{13}$ \\
\hline${ }^{46} \mathrm{Ti}$ & 0.8 & $\mathrm{Av}$ & 2.6 & 308 & 8.6 & 76.1 & 3.6 & \multicolumn{1}{c}{$\theta_{F}$} \\
& & $S$ & 2.6 & 300 & 8.2 & 74.8 & 4.1 & 19.5 \\
& 2.1 & $\mathrm{Av}$ & 2.8 & 345 & 23.9 & 79.0 & 4.2 & 14.4 \\
& & $S$ & $2.9 \pm 0.2$ & $336 \pm 5$ & $22.7 \pm 0.5$ & $77.7 \pm 0.6$ & $4.8 \pm 0.2$ & $15.4 \pm 0.7$ \\
${ }^{166} \mathrm{Er}$ & 2.1 & $\mathrm{Av}$ & 3.6 & 2117 & 79 & 295 & 3.8 & 15.9 \\
& & $S$ & $3.5 \pm 0.3$ & $2070 \pm 28$ & $76 \pm 2$ & $293 \pm 2$ & $4.2 \pm 0.3$ & $17.0 \pm 0.6$ \\
\hline \hline
\end{tabular}


increase of $\mathbf{N}_{\pi}$ for the case Av as compared to SOS. This effect was already pointed out in the discussion of the impact parameter averaged ensemble.

The inclusive cross sections $d N / d E$ for random orientations (Fig. 4) do not show a big difference to the case of polarized nuclei, Fig. 2. There are slightly larger discrepancies between the spherical and randomly oriented deformed cases. Also at the lower beam energy no significant effects are observed. The differences between the cases $S$ and Av become even smaller with decreasing energy.

If the standard deviation of the distributions of all observables is taken into account, we find an unexpected decrease of the widths of the distributions by about $10 \%$. This indicates that although we have less spectators in the reaction with deformed nuclei, a large forward-peaked background of nonthermalized particles is the dominant contribution. On the other hand, the particles having suffered a large number of collisions, which implies large fluctuations, are of minor importance compared to reactions of two spherical nuclei.

\section{CONCLUSIONS}

The aim of this paper is to point out the influence of different initializations of nuclear shapes with strong quadrupole deformations for a rather light and a heavy system within the experimentally available range of relativistic projectile energies. We interpret the distinct differences between $\mathrm{HOH}$ and $\mathrm{BOB}$ collisions in terms of the increased stopping length. The tendency of the changes and their magnitude can be estimated if more complicated shapes like an additional hexadecupole deformation or other special orientations of the incoming nuclei would be considered. In the picture of the intranuclear cascade simulation, the present results should also hold approximately for heavier systems.

We have found a non-negligible influence of the relative orientation in a central collision of deformed nuclei, which could provide information on how much of the initial state properties survive a high energy heavy-ion collision. On the other hand, impact parameter averaging and random orientations yield much smaller differences as a result of the averaging. The distributions of the observables become slightly broader. For the situation of simultaneously requiring random orientations of the incoming nuclei and impact parameter averaging the former distinct differences practically disappear. The fluctuations in observables do not increase as a result of the ensemble average. Therefore in the present day experiment two incoming spherical nuclei represent a reasonable approximation for the description of relativistic heavy ion collisions, if nonpolarized beams and targets are used.

\section{ACKNOWLEDGMENTS}

The Joint Institute for Heavy Ion Research has as member institutions the University of Tennessee, Vanderbilt University, and the Oak Ridge National Laboratory; it is supported by the members and by the Department of Energy through Contract No. BE-AS05-76ER0-4936 with the University of Tennessee. This work was supported by the Bundesministerium für Forschung und Technologie and by the Gesellschaft für Schwerionenforschung.
"Permanent address: Institut für Theoretische Physik, Universität Frankfurt, Frankfurt, Federal Republic of Germany.

${ }^{1}$ A. Rosenhauer, J. A. Maruhn, H. Stöcker, and W. Greiner, Phys. Lett. 159B, 239 (1985).

${ }^{2}$ H. A. Gustafsson, H. H. Gutbrod, B. Kolb, H. Löhner, B. Ludewigt, A. M. Poskanzer, T. Renner, H. Riedesel, H. Ritter, A. Warwick, F. Weik, and H. Wiedman, Phys. Rev. Lett. 52, 1590 (1984); R. Renfordt, D. Schall, R. Bock, R. Brockmann, J. Harris, A. Sandoval, R. Stock, H. Stroebele, D. Bangert, W. Rauch, G. Odyniec, H. Pugh, and L. Schroeder, Phys. Rev. Lett. 53, 763 (1984).

${ }^{3}$ W. Scheid, H. Müller, and W. Greiner, Phys. Rev. Lett. 32, 741 (1974); H. Stöcker, J. A. Maruhn, and W. Greiner, ibid. 44, 725 (1980); G. Buchwald, G. Graebner, J. Theis, J. A. Maruhn, W. Greiner, and H. Stöcker, ibid. 52, 1594 (1984).

4J. Cugnon, Phys. Rev. C 22, 1885 (1980); J. Cugnon, T. Mizutani, and J. Vandermeulen, Nucl. Phys. A352, 505 (1981); G. Bertsch and J. Cugnon, Phys. Rev. C 24, 2514 (1981).

${ }^{5}$ H. J. Erasmuspe, Nucl. Phys. A101, 138 (1967).
${ }^{6}$ C. Bemis, P. Stelson, F. McGowan, W. Milner, J. Ford, R. Robinson, and W. Tuttle, Phys. Rev. C 8, 1934 (1973).

7J. Cugnon and D. L'Hote, Nucl. Phys. A397, 519 (1983).

${ }^{8} \mathrm{We}$ would like to point out that here we use the bound version of the Cugnon cascade which produces a zero degree peaking in the flow angular distributions $d N / d \cos \theta$. It should be clarified that the large flow angles reported in the previous reference are the result of an instability of the colliding nuclei. This has first been pointed out in $\mathbf{H}$. Kruse, J. Jacak, and $\mathbf{H}$. Stöcker, Phys. Rev. Lett. 54, 289 (1985), and was recently substantiated in the article by J. J. Molitoris, H. Stöcker, H. A. Gustafsson, J. Cugnon, and D. L'Hote, Phys. Rev. C 33, 867 (1986). In these latter articles it is emphasized that agreement with experimental data can only be obtained by introducing explicitly a strong short range repulsion into microscopic theory in analogy to the compressional pressure in the fluid dynamical model.

${ }^{9}$ N. L. Balazs, B. Schürmann, K. Dietrich, and L. P. Csernai, Nucl. Phys. A424, 605 (1985). J. Kapusta and D. Strottman, Phys. Lett. 106B, 33 (1981). 


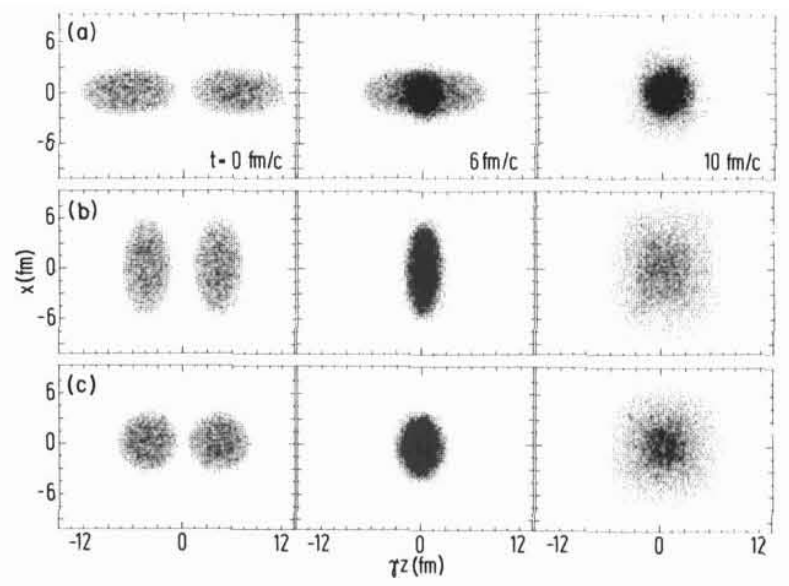

FIG. 1. Illustration of the compression and expansion stage in a central collision. The density at different reaction times as indicated is plotted for the three constellations. The head on head collision corresponds to (a), the belly on belly orientation to (b), and the reaction of two spherical nuclei $\left({ }^{46} \mathrm{Ti}+{ }^{46} \mathrm{Ti}\right.$ at 2.1 $\mathrm{GeV} /$ nucleon) is illustrated in (c). 